\title{
Efficiency performance effect of TiO2 thickness deposited on FTO coated glass photoanode
}

\begin{abstract}
This paper presents the fabrication and thickness analysis of $\mathrm{TiO} 2$ on Fluorine-doped Tin Oxide (FTO) glass for Dye-Sensitized Solar Cell (DSSC). The TiO2 layer was deposited on the cleaned FTO glass using doctor blade technique and the thickness of the $\mathrm{TiO} 2$ were controlled by with different layers of adhesive tape. This results showed that the two-layer with thickness $15.09 \mu \mathrm{m}$ of adhesive tape achieved the highest efficiency of $4.73 \%$, and followed by three-layer and one-layer at $3.64 \%$ at $2.32 \%$ respectively.
\end{abstract}

Keyword: FTO; Dye-sensitized solar cell; TiO2; Thickness; Photoanode 